\title{
Pedicled Island Latissimus Dorsi Myocutaneous Flap in Reconstruction of Complex 3D Head \& Neck Defects after Tumor Ablation: Refinement of Flap Insetting Techniques in Selected Patients
}

\author{
AHMED S. HWEIDI, M.D.*; TAREK ELDAHSHOURY, M.Sc.**; MOHAMMED ALEEM, M.D.*** and \\ SAMER A. IBRAHIM, M.D.*** \\ The Department of Plastic \& Reconstructive Surgery, Faculty of Medicine, Ain Shams University*, \\ The Department of Plastic Surgery, Salisbury District Hospital, UK** and The Department of Otolaryngology (ENT), \\ Faculty of Medicine, Ain Shams University***
}

\begin{abstract}
Introduction: Nowadays free tissue transfer is considered the golden slandered in reconstruction of major defects in head and neck regions. Pedicled flaps, however, still have role in high risk patients. Pedicled island LD myocutanious flap can provide a massive amount of tissue that can be utilized and manipulated to reconstruct complex 3D defects with minimal donor site morbidity. Although has been described long time ago, the literature is very deficient regarding methods and techniques to manipulate the flap during transfer and insetting to fit complex two or three layered 3D reconstruction. The aim of this study is to describe our techniques in an algorithmic pattern within the context of our case series.
\end{abstract}

Methods: In this retrospective study we present 32 patients underwent island LD myocutaneous flap for reconstruction of complex head and neck defects (19 patients underwent partial or total pharyngeal reconstruction \pm neck skin, and 13 patients underwent lower face \pm mandibular reconstruction with intra oral extension). Average age was 56 years and selection criteria for this technique includes; depleted neck vessels from previous surgery, frozen neck after pre-operative radiation together with high risk patients. Technical consideration in flap design and in-setting were reported in details according to the site of reconstruction.

Results: We have a single mortality in post-operative period in a medically morbid patient. No whole flap failure in all patients. 2 patients $(6.3 \%)$ had partial flap loss. Eight patients $(25 \%)$ had other complications like fistula, wound dehiscence and donor site problems. Recurrence happened in 3 patients. Over all complication rate is $31 \%$. Donor site and wound related complications were treated conservatively however other complications such as flap vascular insult, partial necrosis and delayed fistulas were treated surgically $(9.3 \%)$.

Conclusion: The island subcutaneous LD myocutanious flap is a very useful tool in reconstruction of complex 3D head and neck defects in selected patients. The rate of complications are comparable with free tissue transfer when appropriate techniques are used.

Key Words: Pedicled island - Latissimus dorsi-Myocutaneous flap - 3D head \& neck defects - Tumour ablation - Flap insetting techniques.

\section{INTRODUCTION}

Head and neck reconstruction is a continuously developing area of plastic surgery. Usually a three dimensional defect develops after a massive tumor excision which is itself a challenge for plastic surgeons. Added to the complexity, the aesthetic requirement of the reconstructed region, the neck scarring due to previous dissection and the radiated bed sometimes. The gold standard solution for these defects is the microsurgical free tissue transfer which can be of different components of tissue and/or in several stages [1]. Nevertheless, few conditions could render some cases difficult for free tissue transfer [2]. Among these conditions are the previous radiated or scarred vessels, the fragile patient who is not fit for a long time anesthesia and salvage procedures as in tumor recurrence or early or late failure of a free flap which often highlight a problem in recipient vessel selection [3]. In these particular situations, the pedicled Latissimus Dorsai (LD) myo-cutanous, with its enormous tissue, flap still finds its own indications and applications [4-6]. Although this flap has been described for decades ago [7], technical refinements of the original description are limited because it has stepped back in the era of microsurgery. Another reason for the refinements limitations are is the lake of adequate planning when the flap is used as a lifeboat to solve a really complicated problem in a timely fashion. We hereby reports a case series with description of some refinements which can aid optimization of this technique when indicated.

\section{PATIENTS AND METHODS}

All patients were seen by the head and neck cancer multi-disciplinary team at Ain Shams Uni- 
versity Hospitals. The team consists of ENT surgeons, Plastic surgeons, pathologists, radiologists and oncologists. All cases underwent surgery in the same institute from March 2015 till March 2019. In this case series we present thirty two patients. Twenty six were males and six females. The mean age was 56 (ranging from 35 years to 72 years) (Table 1 ).

Although free tissue transfer has been the mainstay for reconstruction of complex 3D head and neck defects in our practice, in these selected cases we used the island latissimus dorsi myocutaneous flap. We believe this technique still has a role in cases where results of free tissue transfer and microvascular anastomosis is not promising. Selection criteria for using this technique includes patients with recurrence or residual lesions where neck vessels were depleted from previous surgery, Frozen neck with significant fibrosis resulted from preoperative radiotherapy and patients with significant medical comorbidities even in primary tumors. Nineteen cases had recurrent or residual tumors and 13 cases were primary tumors in high risk patients (Table 1).

Table (1): Demography and surgical plan in the study population.

\begin{tabular}{|c|c|c|c|c|c|}
\hline $\begin{array}{l}\text { Patient } \\
\text { No. }\end{array}$ & Gender & Age & $\begin{array}{l}\text { Pathology/ } \\
\text { tumor status \& site }\end{array}$ & Excision/defect & $\begin{array}{l}\text { Strategy of } \\
\text { flap insetting }\end{array}$ \\
\hline 1 & M & 45 & SCC, primary, L & - Neck skin + total laryngectomy \& pharyngeoplasty & 1 layer \\
\hline 2 & M & 55 & $\mathrm{SCC}$, recurrent, $\mathrm{L}$ & - Neck skin + total laryngectomy \& pharyngeoplasty & 1 layer \\
\hline 3 & M & 48 & $\mathrm{SCC}$, residual, $\mathrm{L}$ & - Neck skin + total laryngectomy \& pharyngeoplasty & 1 layer \\
\hline 4 & M & 57 & $\mathrm{SCC}$, primary, $\mathrm{L}$ & - Neck skin + total laryngectomy \& pharyngeoplasty & 1 layer \\
\hline 5 & M & 59 & $\mathrm{SCC}$, primary, $\mathrm{H}$ & - Neck skin + total laryngectomy \& pharyngeoplasty & 1 layer \\
\hline 6 & M & 43 & $\mathrm{SCC}$, recurrent, $\mathrm{L}$ & - Neck skin + total laryngectomy \& pharyngeoplasty & 1 layer \\
\hline 7 & $\mathrm{~F}$ & 65 & $\mathrm{SCC}$, recurrent, $\mathrm{L}$ & - Neck skin + total laryngectomy \& pharyngeoplasty & 1 layer \\
\hline 8 & M & 65 & $\mathrm{SCC}$, residual, $\mathrm{H}$ & - Neck skin + total laryngectomy \& pharyngeoplasty & 1 layer \\
\hline 9 & M & 41 & SCC, primary, L & - Neck skin + total laryngectomy \& pharyngeoplasty & 1 layer \\
\hline 10 & M & 60 & $\mathrm{SCC}$, recurrent, $\mathrm{L}$ & - Neck skin + total laryngectomy \& pharyngeoplasty & 1 layer \\
\hline 11 & M & 56 & SCC, primary, L & - Neck skin + total laryngectomy \& pharyngeoplasty & 1 layer \\
\hline 12 & M & 48 & $\mathrm{SCC}$, recurrent, $\mathrm{L}$ & - Neck skin + total laryngectomy \& pharyngeoplasty & 1 layer \\
\hline 13 & M & 61 & $\mathrm{SCC}$, recurrent, $\mathrm{L}$ & - Neck skin + total laryngectomy \& pharyngeoplasty & 1 layer \\
\hline 14 & M & 47 & SCC, recurrent, $\mathrm{L}$ & - Neck skin + total laryngectomy \& pharyngeoplasty & 1 layer \\
\hline 15 & M & 53 & SCC, primary, $\mathrm{H}$ & - Neck skin + total laryngectomy \& pharyngeoplasty & 1 layer \\
\hline 16 & M & 70 & SCC, residual, L & $\begin{array}{l}\text { - Neck skin + total laryngectomy \& pharyngeoplasty } \\
\text { (flap forms ant wall) }\end{array}$ & 1 layer \\
\hline 17 & $\mathrm{~F}$ & 62 & SCC, recurrent, L & $\begin{array}{l}\text { - Neck skin + total laryngectomy \& pharyngeoplasty } \\
\text { (flap forms ant wall) }\end{array}$ & 1 layer \\
\hline 18 & M & 35 & SCC, primary, L & - Neck skin + total laryngeo - pharyngectomy & 3 layers \\
\hline 19 & M & 47 & $\mathrm{SCC}$, recurrent, $\mathrm{L}$ & - Neck skin + total laryngeo - pharyngectomy & 3 layers \\
\hline 20 & M & 57 & $\mathrm{SCC}$, primary, LF/N & - WLE/lower face \& neck skin & 1 layer \\
\hline 21 & M & 55 & $\mathrm{SCC}$, primary, LF/N & - WLE/lower face \& neck skin & 1 layer \\
\hline 22 & $\mathrm{~F}$ & 59 & $\mathrm{BCC}$, recurrent, $\mathrm{LF} / \mathrm{MF} / \mathrm{IO}$ & - WLE/lower \& midface skin + IO & 2 layers \\
\hline 23 & $\mathrm{~F}$ & 68 & SCC, primary, LF/M/IO & $\begin{array}{l}\text { - WLE/lower face skin \& lat. Segmental } \\
\text { mandiblectomy + IO }\end{array}$ & $\begin{array}{l}2 \text { layers }+ \\
\text { recon plate }\end{array}$ \\
\hline 24 & M & 43 & $\mathrm{SCC}$, primary, LF/M/IO & $\begin{array}{l}\text { - WLE/lower face skin \& segmental mandiblectomy } \\
+ \text { IO }\end{array}$ & $\begin{array}{l}2 \text { layers }+ \\
\text { recon plate }\end{array}$ \\
\hline 25 & M & 64 & SCC, primary, LF/M/IO & $\begin{array}{l}\text { - WLE/lower face skin \& marginal mandiblectomy + } \\
\text { IO }\end{array}$ & 2 layers \\
\hline 26 & M & 67 & Basi-Sq, recurrent, LF/M/IO & $\begin{array}{l}\text { - WLE/lower face skin\& ant. segmental mandiblectomy } \\
+ \text { IO }\end{array}$ & $\begin{array}{l}2 \text { layers }+ \\
\text { rib }\end{array}$ \\
\hline 27 & M & 47 & $\mathrm{SCC}$, recurrent, $\mathrm{LF} / \mathrm{N}$ & - WLE/lower face \& neck skin & 1 layer \\
\hline 28 & M & 49 & SCC, recurrent, LF/N/IO & - WLE/lower face \& neck skin + IO & 2 layers \\
\hline 29 & $\mathrm{~F}$ & 60 & BCC, recurrent, LF/M/IO & $\begin{array}{l}\text { - WLE/lower face skin \& marginal madiblectomy + } \\
\text { IO }\end{array}$ & $\begin{array}{l}2 \text { layers }+ \\
\text { recon plate }\end{array}$ \\
\hline 30 & M & 64 & SCC, primary, LF/N/IO & - WLE/lower face and neck skin + IO & 2 layers \\
\hline 31 & $\mathrm{~F}$ & 72 & SCC, recurrent LF/M/IO & $\begin{array}{l}\text { - WLE/lower face skin \& marginal mandiblectomy } \\
+ \text { IO }\end{array}$ & 2 layers \\
\hline 32 & M & 70 & SCC, recurrent, LF/IO & - WLE, lower face skin + IO & 2 layers \\
\hline \multirow{6}{*}{\multicolumn{4}{|c|}{$\begin{array}{l}\text { *M }: \text { Indicates male. } \\
\mathrm{F} \quad \text { : Indicates female. } \\
\mathrm{SCC}\end{array}$}} & MF : Indicates Midface. & \\
\hline & & & & : Indicates Neck. & \\
\hline & & & & : Indicates Mandible. & \\
\hline & & & & IO : Indicates Intra Oral extension. & \\
\hline & & & & Recon: Indicates Reconstruction. & \\
\hline & & & & WLE : Indicates Wide Local Excision. & \\
\hline
\end{tabular}


All patients had their workup done including; blood tests, CAT scan with contrast to assess tumor extension and lymph nodes involvement. Incisional biopsies were done in all cases to confirm pathology, even endoscopic or directly according to site of the tumor. All resections and neck dissections were done by the ENT team in joint with Plastic surgery team. Seventeen patients underwent total laryngectomy and pharyngeoplasty followed by reconstruction of neck skin defect by 1 layer flaps, 2 patients underwent total laryngeo- paharngectomy followed by reconstruction of the pharengeo- esophageal tube and the neck skin by 3 layered flaps. Thirteen patients underwent wide local excision of facial and neck skin involved \pm intra oral extension; with or without madiblectomy (either segmental or marginal). Four patients underwent mandibular reconstruction ( 3 by reconstruction plate. and 1 by composite rib within the LD flap). In all 13 patients the LD flap was used to reconstruct the 3D defect either in single or double layers (10 cases double layer reconstruction and 3 cases single layer reconstruction) (Table 1). Twenty three patient out of the $32(71.9 \%)$ underwent neck dissection by the ENT team on the same surgery; 18 underwent functional neck dissection, 4 underwent selective neck dissection and 1 patient underwent modified radical neck dissection.

Surgical techniques: Planning and pre-operative marking were done while the patient in standing position, is possible, after accurate measurement of the expected defect bearing in mind the three dimensional nature of the defect that prompt us to use multi-layer technique in insetting of the flap if needed. We start by identifying the LD muscle borders; anterior border is detected and marked on the posterior axillary fold by forceful contraction of the muscle, posterior border is the back midline, superior border just pass transversely over the angle of the scapula, and inferior border is the posterior iliac crest. The thoracodorsal vessels are marked just medial to the upper part of the anterior border and the entry point is marked as well (usually $8 \mathrm{~cm}$ from the apex of posterior axillary fold) the skin paddle is designed obliquely along the anterior border of the muscle usually in the lower half to insure good arc of rotation without tension on the pedicle. The lower extension of the skin paddle, however didn't cross a point $3 \mathrm{~cm}$ above the iliac crest, as the skin perfusion beyond this point is usually not very reliable. In multi layered reconstruction the skin paddle is divided according to the defect by lines (1-2cm in thickness) that resemble areas for de-epithelization that separate different layers and cavities in the planed $3 \mathrm{~d}$ reconstruction (Fig. 1).
After performing the excision by the ENT surgeons in supine position, the wound is covered and the patient is put in lateral decubitus and redraped. The incision is made along the borders of the planed skin paddle circumferentially and extended superiorly towards the axilla. Dissection and identification of the 4 borders of the LD muscle is then performed with meticulous homeostasis. The LD muscle is then separated from the underlying serratus anterior muscle. Finally the thoracodorsal pedicle is identified under loups magnification and tracked and dissected meticulously up to its take of point from the subscapular vessels. Other branches are tied and divided to allow full mobilization and freeing of the pedicle; this step is done while the assistant open the axilla (position the shoulder in abduction). The LD muscle is then elevated after dividing all origin attachments using monopolar cautery. The insertion of the LD muscle is then identified and separated from the teres major insertion and then divided Fig. (2). It's very important after this step to avoid any tension on the free pedicle; we accomplish this by securing the tendinous part of the LD insertion to the pectoralis major fascia on the anterior axillary fold using two proline suture ( 0 or $2 / 0)$, making sure there is no tension on the pedicle before passing the flap to the head and neck region through a subcutaneous tunnel. Meticulous hemostasis is then performed on the muscle bed and the donor site. Direct closure was performed after undermining in 31 cases among which; 3 flaps required assistance by local Z flaps in closure (Fig. 3) and just 1 patient with very big flap required a small skin graft. Suction drains were used in all donor sites.

\section{Flap insetting:}

Insetting of the flap is done after repositioning the patient in supine position again. The pattern of insetting was determined according to the defect size, shape and site. Insetting was completed in single layer, double layer or triple layer.

Single layered insetting is used in reconstruction of neck skin and provide muscle coverage over the site of pharyngeoplasty (direct closure of the defect on anterior wall of the pharynx) following total laryngectomy with partial pharengectomy. Single layer insetting is used also, in simple 2D facial defects that doesn't entail intraoral extension or mandiblectomy. During the single layered insetting the edge of the defect is undermined only for $1 \mathrm{~cm}$ to allow securing the muscle part of the flap underneath the defect edge with bolster sutures to 
avoid clumping of the muscle part and to provide good water tight closure. This is followed by sub- cutaneous and skin sutures to secure the skin paddle to the edge of the defect (Fig. 4).
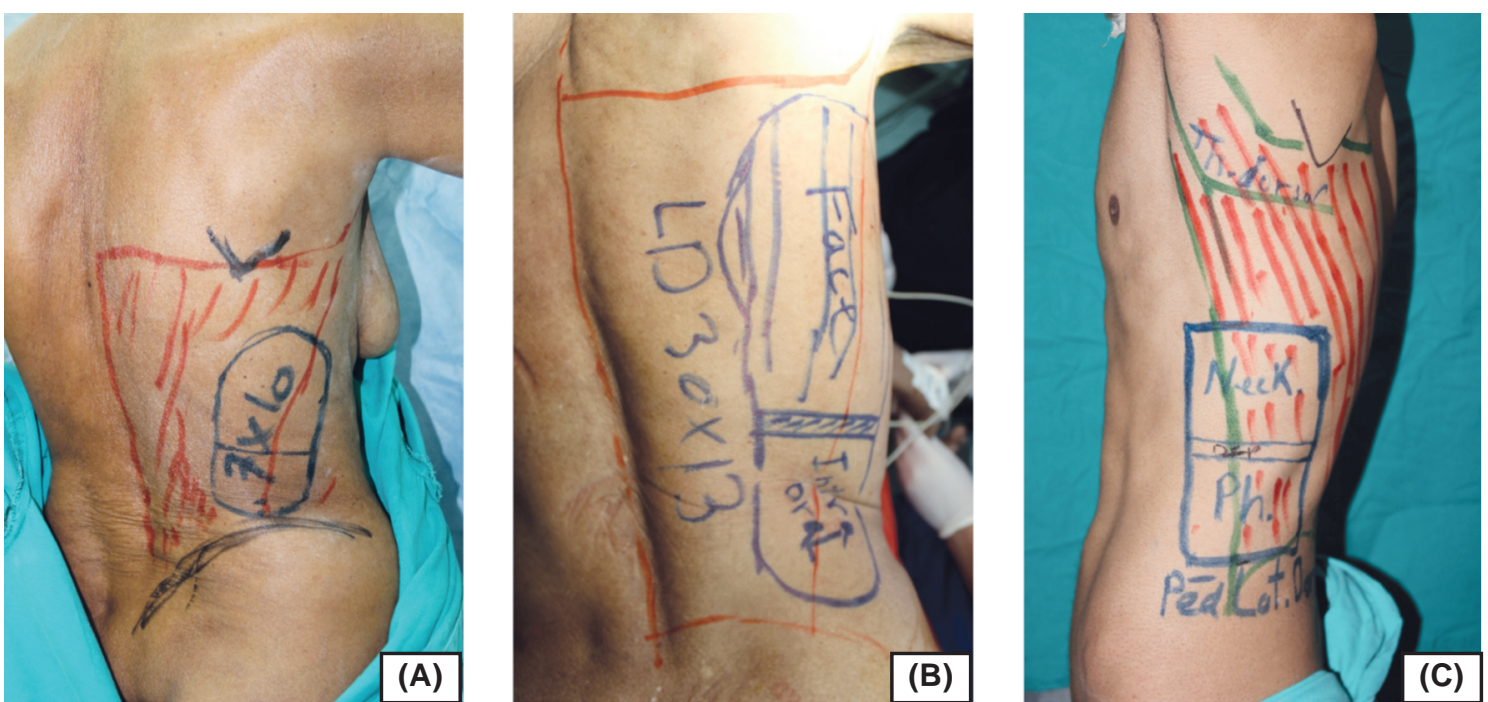

Fig. (1): Design of the skin paddle according to the purpose and the plane of reconstruction. (A) Design for simple single layered flap to cover lower face and neck 2D defect. (B) Double layered flap to cover a 3D lower face defect with intraoral extension, the 2 parts were separated by a $2 \mathrm{~cm}$ strip to be de epithelized to facilitate separation of oral cavity from facial skin. (C)Triple layered flap design for total pharyngeal reconstruction, the distal part was meant to reconstruct the pharyngeal tube (2 layers; anterior and posterior wall) and the proximal part meant to reconstruct the neck skin. The 2 parts are separated by a strip for deepithelization.
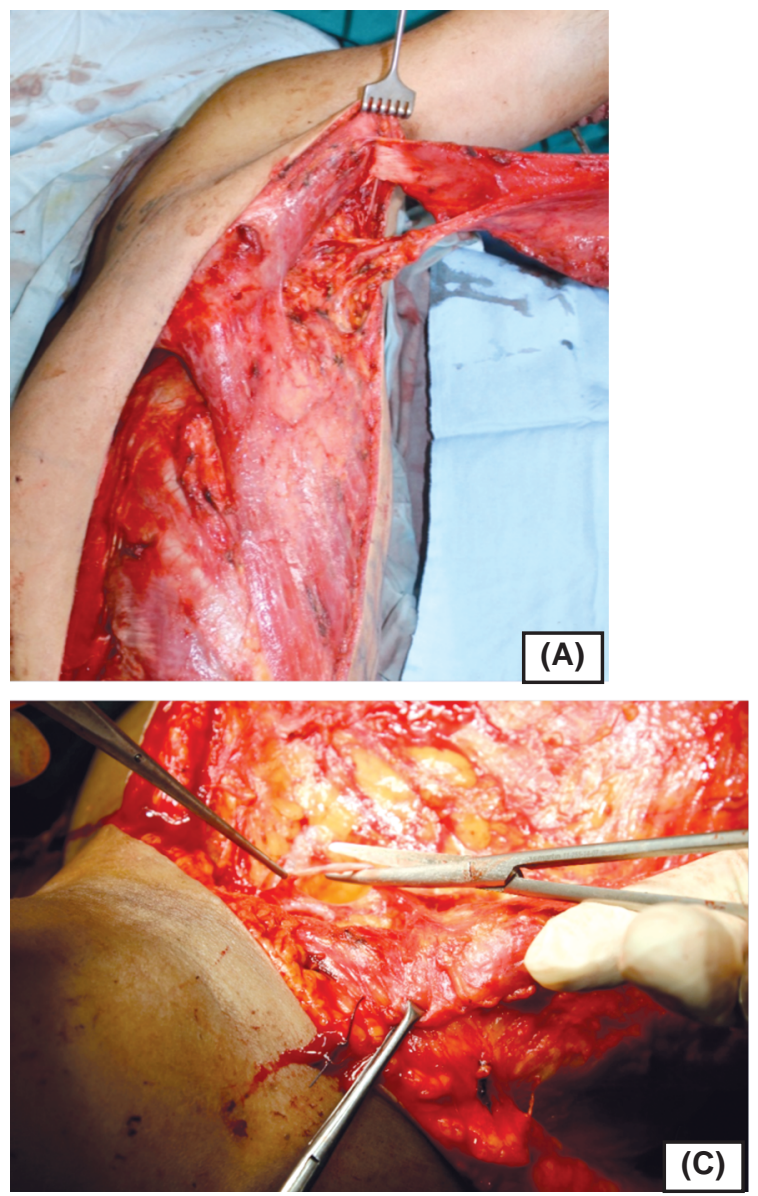

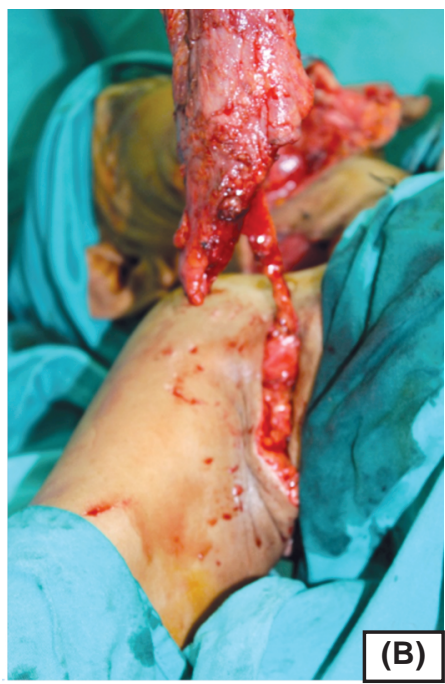

Fig. (2): (A) Dissection of the thoracodorsal pedicle and isolation of the LD tendon up to bicipital groove. (B) Division of the insertion to allow maximum reach of the flap. (C) Microdissection and division of the thoracodorsal nerve to avoid twitches and contractures in the muscle later on. 

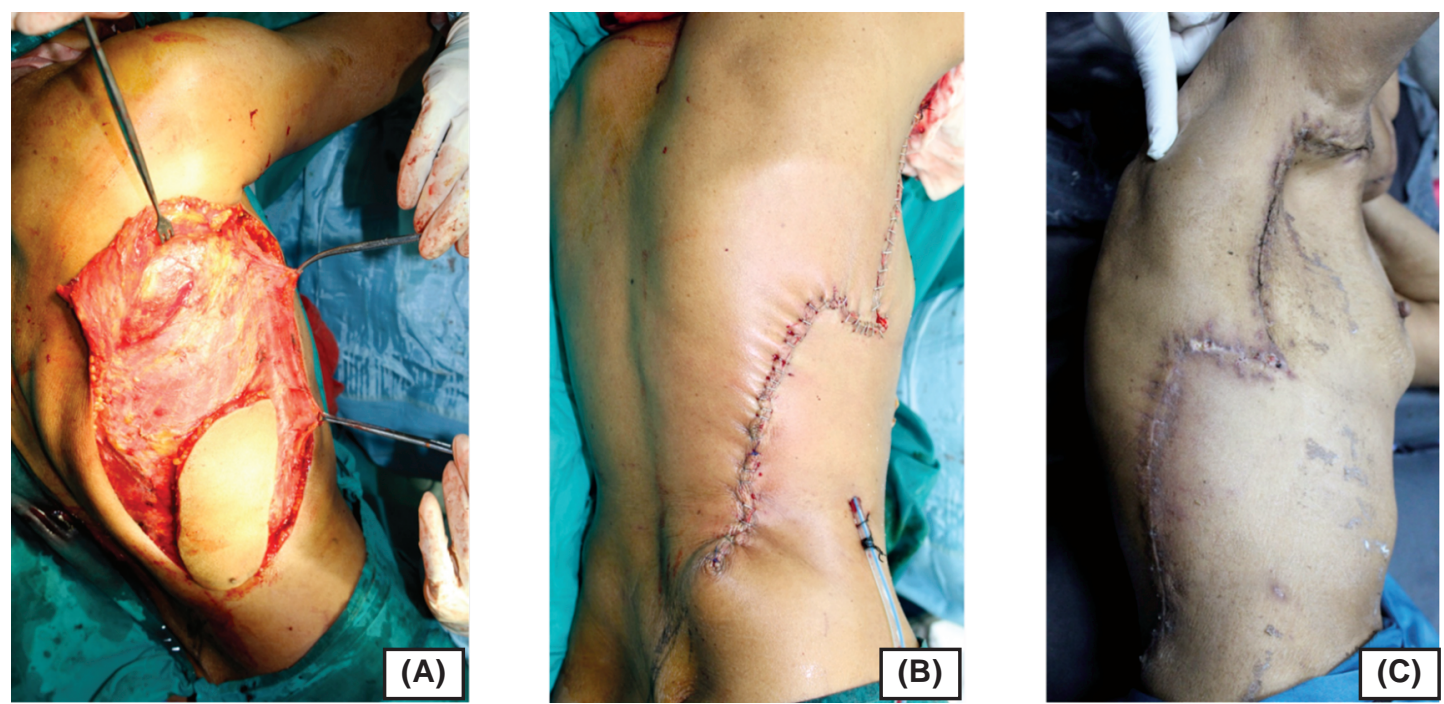

Fig. (3): (A) Undermining of the donor site edges to facilitate direct closure after harvesting of a big flap.

(B) Direct closure facilitated by Z plasty to avoid tension. (C) Complete healing after 3 weeks.
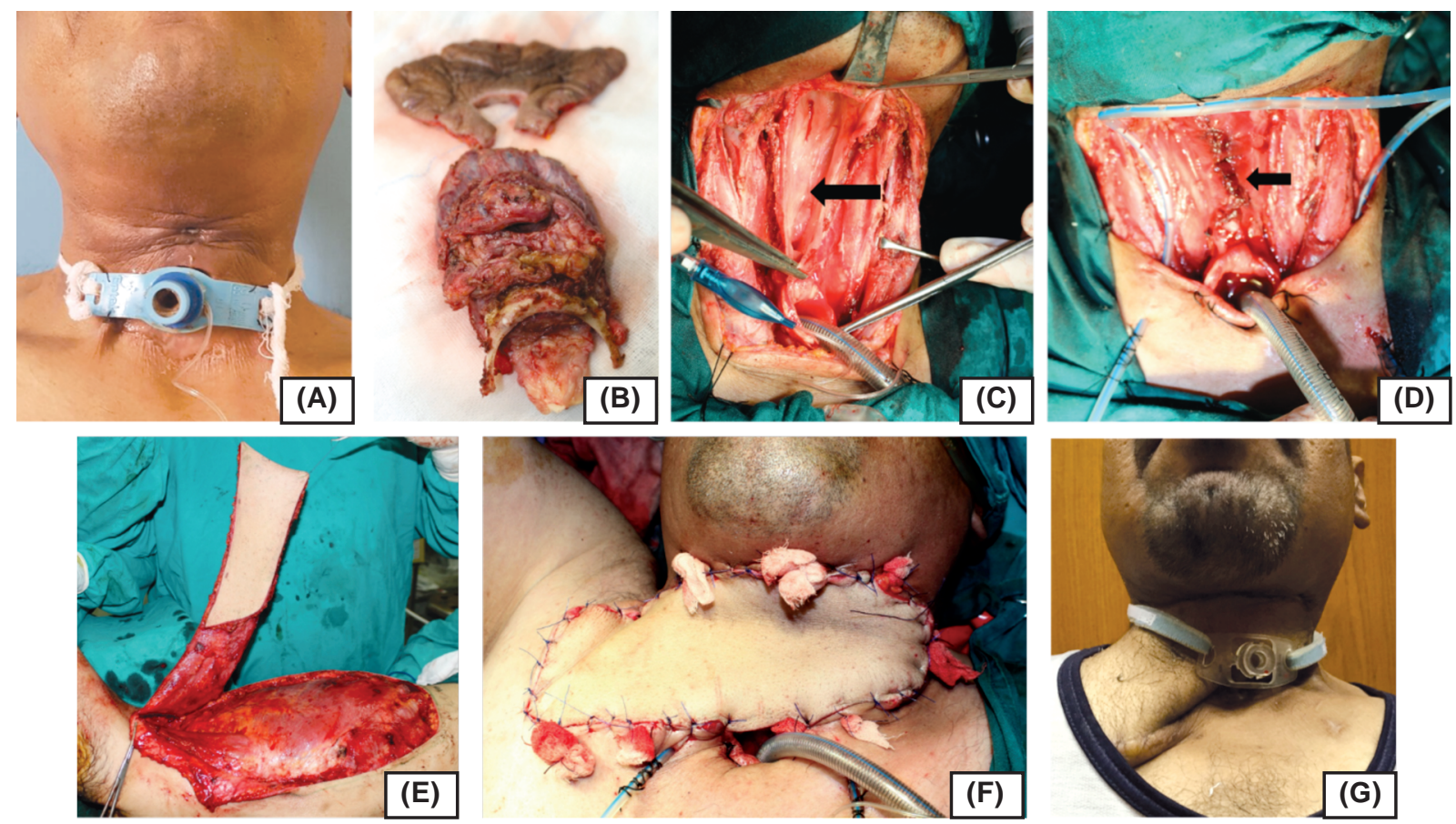

Fig. (4): (Case 4) (A) 57 year old male with primary SCC larynx in a previously radiated neck. (B) The excised specimen consisted of the whole larynx, the anterior wall of the pharynx as a safety margin and the radiated neck skin. (C) Pharyngeal cavity lay open indicated by arrow. (D) Pharyngeplasty in layers tested by methylene blue to insure its water tight. (E) The LD flap after total freeing (F) Insetting of the flap in one layer where the muscle edges were pulled under the defect edges and secured by bolster sutures to avoid skin necrosis.

Double layered insetting is utilized in reconstruction of full thickness 3D defects of facial skin with intraoral extension \pm madiblectomy. In the 2 layered insetting, we secure the distal end of the flap to the edges of the intraoral defect using $3 / 0$ vicryl running sutures (better water tight). Then we de epithelize a $1 \mathrm{~cm}$ strip on the skin paddle. The location of this strip is pre-designed in preoperative marking and can be modified intra operatively to be located between the intra and extra oral portion of the flap. One edge of this de epi- thelized strip is sutured to the mucosa intra orally and the other edge is sutured to the skin extra orally, then the rest of the extra oral portion of the flap is secured similar as in single layered insetting. This de epithelized strip provide robust separation between the 2 layers of the flap and betweein the oral cavity and the skin Fig. (5). In cases where lower lip is excised completely we did not do deepithelization as the line of separation between the intra oral part and the facial skin supposed to be the new lip (Fig. 6). 

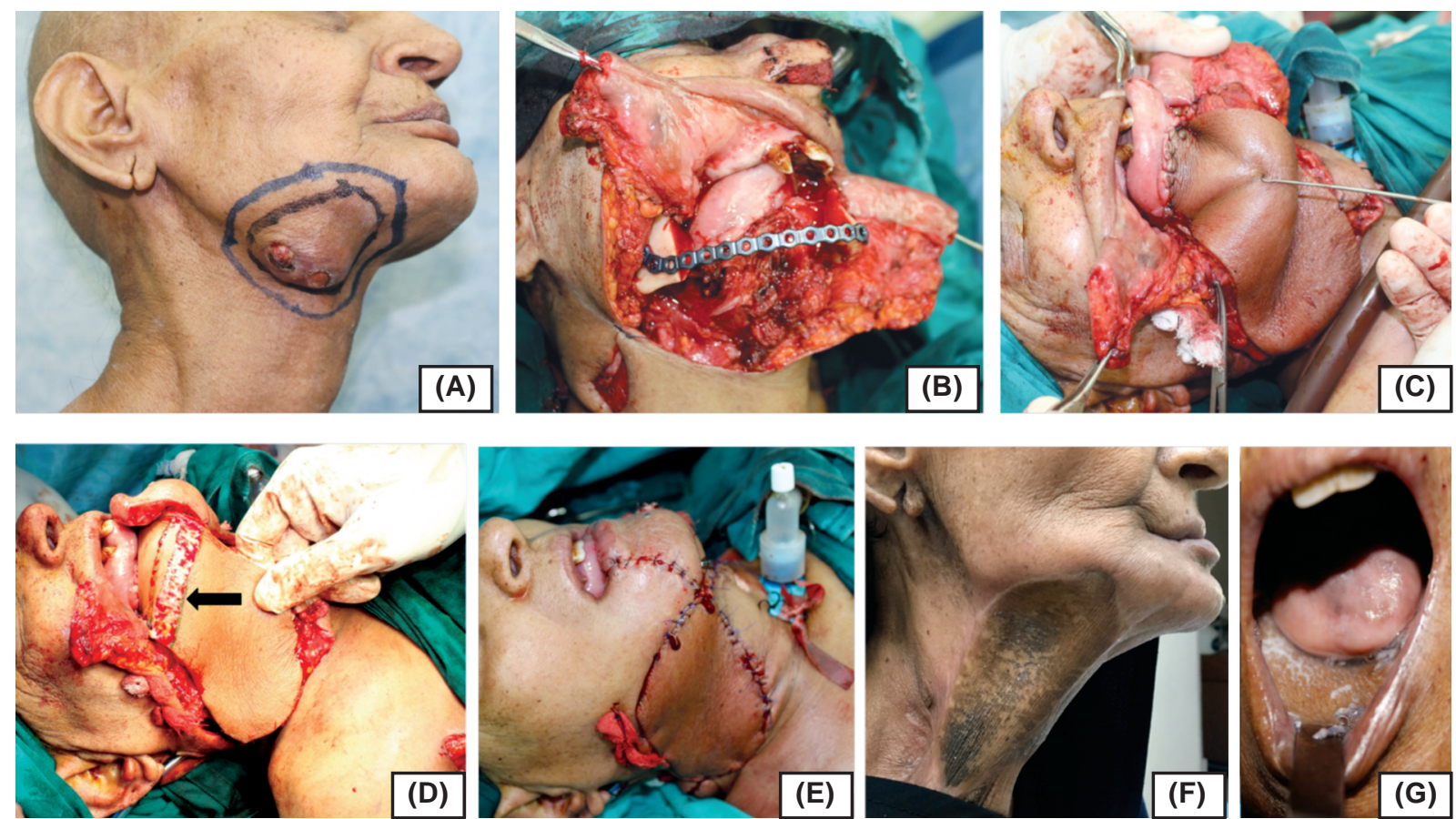

Fig. (5): (Case 23) (A) 68 year old female with intra oral SCC infiltrating the mandible and the lower face and neck skin. (B) Excision in block and reconstruction of mandible by a reconstruction 2.7 plate. (C) Suturing the distal end of the flap to the edges of the intra oral defect to create the $1^{\text {st }}$ layer of insetting (D) De epithelization of a strip of skin paddle to separate the 2 layers of the flap where the distal edge of the de epithelized strip will be sutured in continuity with the edges of intra oral defect, and the proximal edge will be sutured to the face skin defect, the purpose is to separate the oral cavity from the face skin by 2 water tight layers. (E) Insetting of the second layer to cover face and neck skin defect. (F) Complete healing of the $2^{\text {nd }}$ layer after 4 months. $(\mathrm{G})$ Complete healing of the $1^{\text {st }}$ layer creating the floor of the mouth.
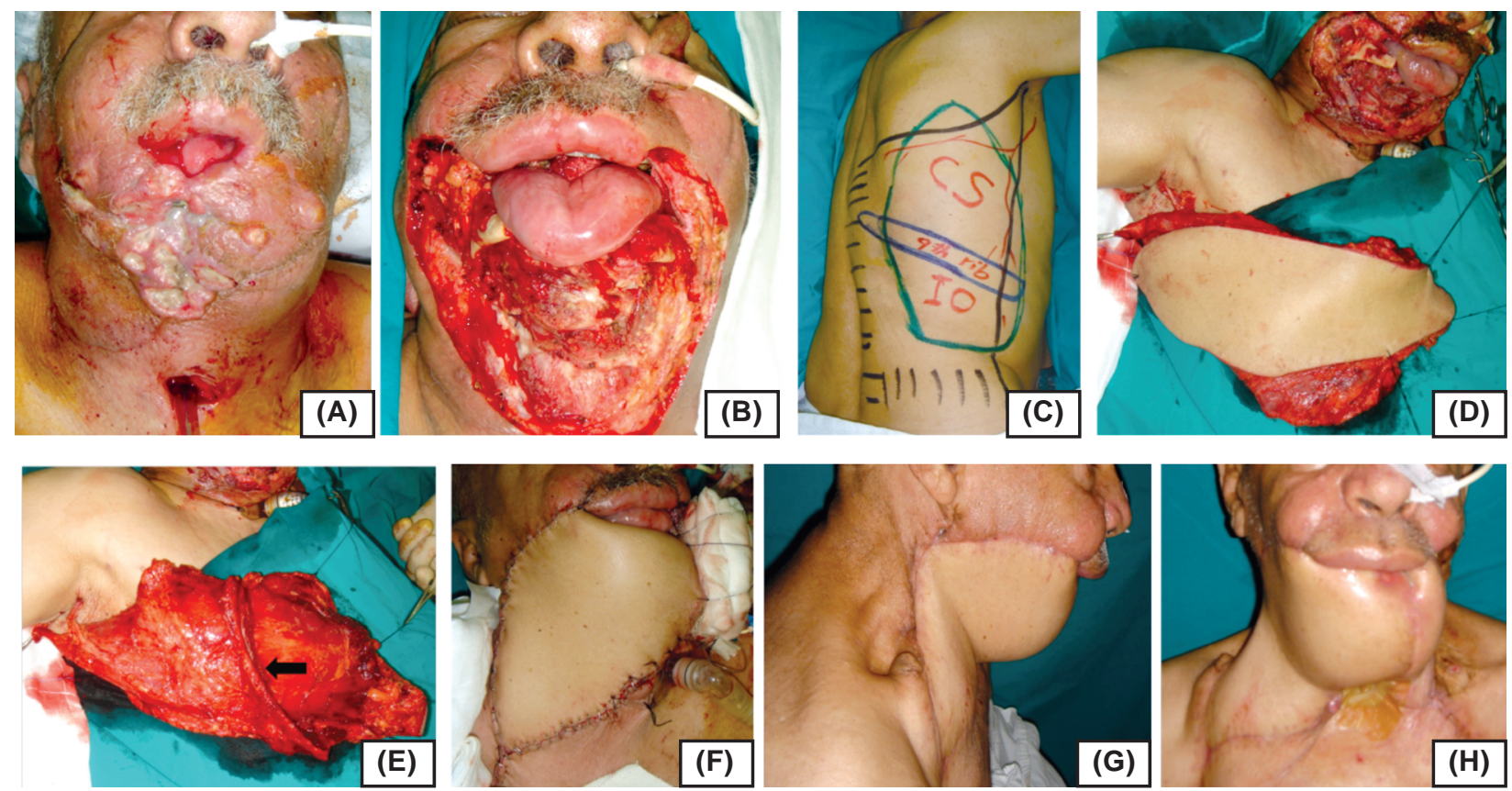

Fig. (6): (Case 26) (A) A 67 year old male suffered from recurrent aggressive basi squamous carcinoma of skin infiltrating the anterior mandible. (B) WLE was done with segmental anterior mandiblectomy. (C) Design of the composite osteomyocutaneous LD flap for composite 3D reconstruction. (D) Although 2 layered insetting of the flap was planned no de epithelization of a strip of the skin paddle was done as the lip couldn't be salvaged in this case. (E) Arrow indicated the $9^{\text {th }}$ rib harvested within the composite flap. (F) Immediate insetting in 2 layers $(\mathrm{G}, \mathrm{H}) 3$ months post-operative. 
Triple layered insetting is utilized to reconstruct neck skin and total pharyngeal (cirumfrantial) reconstruction following total laryngeopharengectomy. The $1^{\text {st }}$ layer reconstruct the posterior pharyngeal wall and it consists of the most distal part of the flap. The $2^{\text {nd }}$ layer is the anterior pharyngeal wall and entails the middle portion of the flap to form a tube with the posterior wall. The upper edge of the tube is anastomosed with oropharynx and the lower end of the tube is anastomosed with the esophageal end. The 3 rd layer is separated from the 2 nd layer by a strip of de epithelization similar to what was done in the 2 layered insetting; where one edge of the de epithelized strip is sutured to the most distal edge of the flap to close the pharyngeal tube laterally. The other edge is sutured to the neck skin on one side of the neck defect and then the rest of the flap (3rd layer) is inset and secured to reconstruct the neck skin (Fig. 7).
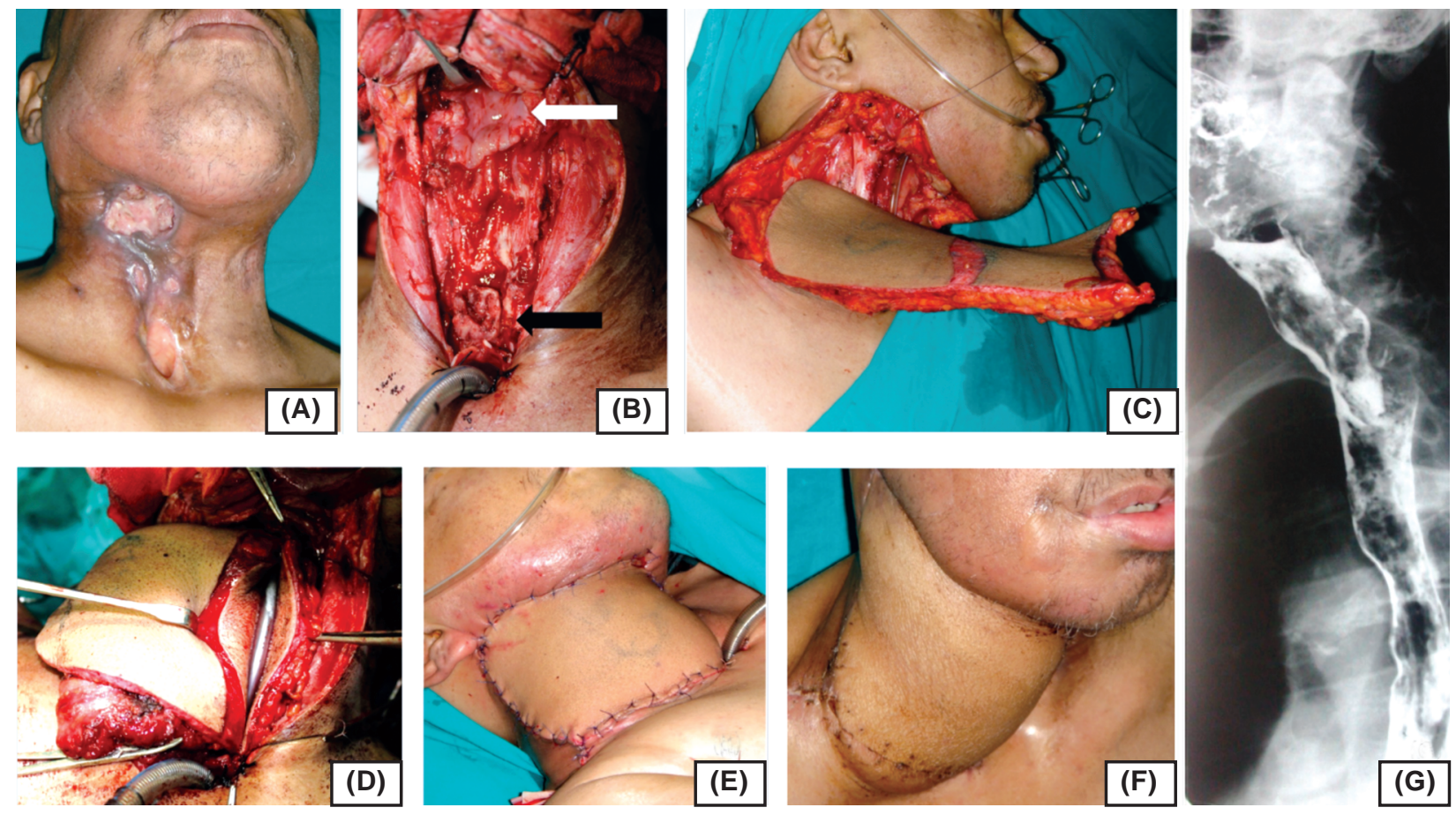

Fig. (7): (Case 19) A 47 yr old male with recurrent SCC larynx and multiple pharyngeo cutaneous fistulas (B) Underwent WLE of neck skin and total pharyngectomy, the white arrow indicates the tongue base and the black arrow indicated the esophagus. (C) The reach of the triple layered flap to the defect with strip de epithelization to separate the reconstructed pharyngeal tube from the reconstructed neck skin. (D) Tubing of the distal portion of the flap around a pre inserted Ryle where the tube was anastomosed superiorly to the remnants of the oropharynx and inferiorly to the esophagus. (E) The most proximal part of the flap, the $3^{\text {rd }}$ layer was used to reconstruct the neck skin. (F) One month post-operative (G) Bariam swallow on the $4^{\text {th }}$ week shows no significant fistulas.

\section{Post-operative care and follow-up:}

Vascularity of the flaps was monitored clinically post-operative together with wound and tracheostomy care. Feeding was maintained immediately post-operative via nasogastric tube in 39 patients and by gastrostomy tube in 3 patients. Oral intake started 10-15 days post-operative guides by healing. We did barium swallow study only in complicated and suspicious patients before starting oral intake. Post-operative radiotherapy started 4-6 weeks after removal of Ryle tube and insuring good healing. Chemotherapy was added in few selective patients based on the oncologist's recommendations. Early and late complications were reported.

\section{RESULTS}

One patient died in early post-operative course in the ICU due to pre-existing cardiovascular compromise. All flaps survived with two partial distal flap losses and were managed by another regional flap (pec. Major) and resetting (advancement of LD). Wound dehiscence happened in 3 patients and healed spontaneously in 2 and required secondary sutures in 1 Fig. (8). Two patients developed minor problems in the donor site; a partial graft loss in one patient and wound dehecence in another patient, both were managed conservatively. 1 patient with total 
circumferential pharyngeal reconstruction suffered from a late high output fistula that was managed surgically by a pectoralis major flap. Three patients suffered from tumor recurrence during the follow-up who were managed in a palliative way as per our multi-disciplinary team. Early flap congestion happened in two patients who underwent tunnel exploration and pedicle resetting in the same day and the flaps survived Fig. (9), (Table 2).
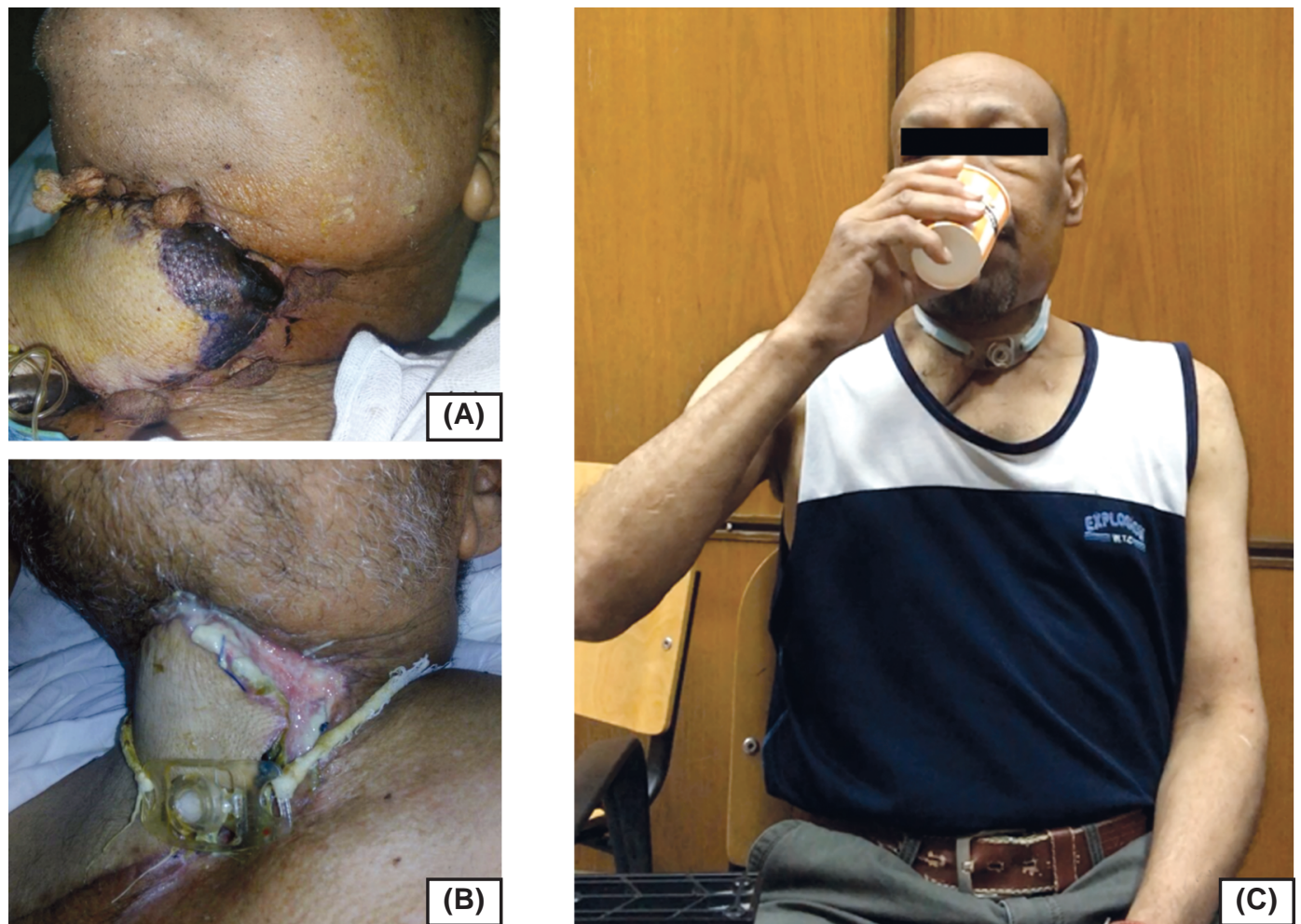

Fig. (8): (A) Vascular compromise of the tip of the skin paddle of LD flap used to reconstruct neck skin after total laryngerctomy and pharyngeoplasty. (B) The healthy LD muscle underneath, that was secured under the defect edges by bolster sutures, saved the patient from another surgery and made the conservative management of wound possible. (C) The patient while drinking water 2 month after surgery after complete healing.
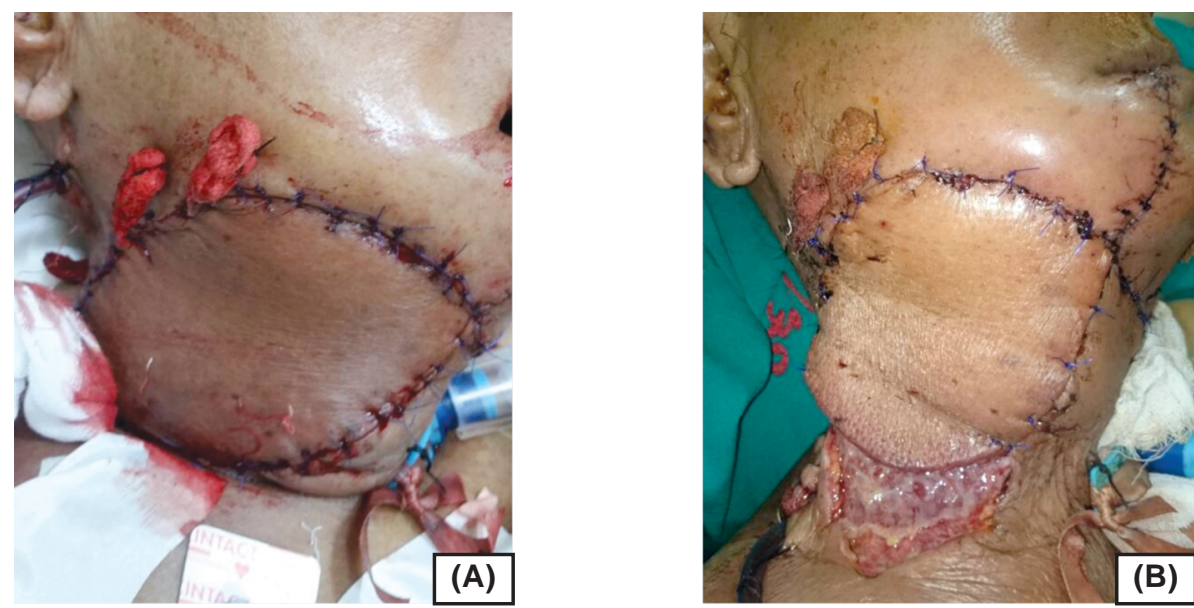

Fig. (9): (Case 23) (A) Early flap congestion was noted few hours after surgery, this was relieved by re-exploration and insure no tension on the pedicle by re insetting of the LD tendon to the pectoral fascia at a nearer point, together with release some stiches. (B) 2 days later with significant improvement in color.

Table (2): Complications and their management.

\begin{tabular}{lccl}
\hline Complications & Number & Percentage & Treatment \\
\hline Partial flap loss & 2 patients & $6.2 \%$ & Resetting/pec-major \\
Wound dehiscence & 3 patients & $9.4 \%$ & 2 conservative/1 secondary sutures \\
Early fistula & 2 patients & $6.2 \%$ & Conservative \\
late pharyngeo-cutanous fistula & 1 patient & $3.1 \%$ & Pec major \\
Donor site complication & 2 patients & $6.2 \%$ & Conservative \\
Total rate of complications & & $31 \%$ & $12.4 \%$ surgical \\
\hline
\end{tabular}




\section{DISCUSSION}

On one hand, most of the defects of the head and neck tumor excision are complicated as the aim to achieve a histological margin of $0.5 \mathrm{~cm}$ and a clear nodes if possible [8]. On the otherhand, head and neck tumors are subjected to immediate reconstruction in most of occasions as a gold standard [9] due to the complexity and position of the defect making the operation inherently longer and morbid. Compared to a large series of free flaps (1000) free flap [10] our total flap failure is $0 \%$ compared to $5.8 \%$, and $6.2 \%$ our partial necrosis to $7.6 \%$ in the free flap series also donor site complications was lower in our series $(6.2 \%$ compared to $8 \%$ in the free flap series).

In our practice, the pedicled LD flap for head and neck reconstruction is usually indicated in complicated patients than free flaps, which in turn gives major and minor complications a bigger chance. However, because the reliability and easiness of this flap, our overall complication rate is comparable to the same flap in the literature [11,12]. Furthermore, the major complications related to the flap itself are very scarce. In the same context, free flaps in these situations will indeed have an unacceptably high rate of complications.

The insetting of the flap in the defect is of vast importance and influences the outcome. There is scarcity of reports of the literature describing the insetting. Watson and John advocated one stage pharyngeal reconstruction and mentioned few details in insetting as a tube and skin cover in a case report [13]. Richard and Vahram have reported 56 cases but most of them were for zygomaticofacial defects with very few details of the insetting using imaginary diagrams rather than pictorial documentation. Few reports followed without focusing on insetting technique $[\mathbf{1 4}, \mathbf{1 5}]$. Thus, in our report we focus on the classification of insetting for various types of defects (one layer for skin or mucosal defect, two layers for double surface defects and three layers in circumferential reconstruction) as described in methods. Fixing the muscle flap underneath the edge of the defects by bolster sutures saved us from facing major complications like fistula. This does not only provide a water seal layer under the skin paddle, but also allows wound dehiscence and minor skin paddle vascular compromise to be managed successfully in a conservative way due to the viable muscle layer underneath.

Denervation of the LD is our usual practice as for breast reconstruction to avoid post-operative twitching [16] which we think decreases the time of healing and the liability of disruption. The Subcutaneous tunneling of the Islanded flap and anchoring of the LD tendon to the Pectoralis major fascia has found to be sufficient and saves us the un-necessary invasive dissection of the transaxillary rout [12] or subclavian rout which might lead to complications as injury of axillary vein and entail osteotomy of clavicle sometimes [17].

Although we have applied this technique due to its advantages in selected patients, it still has some limitations. One of those is the difficulty to monitor the distal portion of the flap which is frequently invisible and discovered only by complications as fistula. Another issue is the technicality to inset a pedicled flap which is more difficult than insetting of a free (mobile) flap before the anastomosis. As a result, a relatively long time is still consumed (average 5 hours for flap harvest and inset) and this is also due to change in position. Finally, the defect size is often over estimated resulting in a bulky flap which is unsafe for debulking at least in the same session.

In Conclusion: The Island pedicled LD flap for major $3 \mathrm{~d}$ head and neck reconstruction is still a lifeboat for difficult cases when carefully planned beforehand and meticulously applied to get the best of its benefits.

\section{REFERENCES}

1- Wong C. and Fu-Chan W.: "Microsurgical free flap in head and neck reconstruction". Head \& Neck, 32.9: 123645,2010 .

2- Singh B., et al.: "Factors associated with complications in microvascular reconstruction of head and neck defects." Plastic and reconstructive surgery, 103.2: 403-11, 1999.

3- Yazar I. and Sukru H.: "Selection of recipient vessels in microsurgical free tissue reconstruction of head and neck defects". Microsurgery: Official Journal of the International Microsurgical Society and the European Federation of Societies for Microsurgery, 27.7: 588-94, 2007.

4- Rigby A., Matthew H. and Richard E.: "Regional flaps: A move to simpler reconstructive options in the head and neck". Current opinion in otolaryngology \& head and neck surgery, 22.5: 401-6, 2014.

5- Ong H., Shan T. and Chen P.Z.: "The pedicled latissimus dorsi myocutaneous flap in head and neck reconstruction". Oral and Maxillofacial Surgery Clinics, 26.3: 427-34, 2014.

6- Novakovic D., et al.: "Salvage of failed free flaps used in head and neck reconstruction". Head \& Neck Oncology, 1.1: 33, 2009 .

7- Quillen C.G. Shearin J.C. and Nicholas G.: "Use of the latissimus dorsi myocutaneous island flap for reconstruction in the head and neck area: Case report". Plastic and Reconstructive Surgery, 62.1: 113-7, 1978. 
8- Hinni A., Michael L., et al.: "Surgical margins in head and neck cancer: A contemporary review". Head \& neck, 35.9: 1362-70, 2013.

9- Eckardt A. and Konstantinos F.: "Microsurgical reconstruction in the head and neck region: An 18-year experience with 500 consecutive cases". Journal of CranioMaxillofacial Surgery, 31.4: 197-201, 2003.

10- Pohlenz P., et al.: "Microvascular free flaps in head and neck surgery: Complications and outcome of 1000 flaps". International Journal of Oral and Maxillofacial Surgery, 41.6: 739-43, 2012.

11- Barton F.E., Thomas E. Spicer and H. Steve Byrd: "Head and neck reconstruction with the latissimus dorsi myocutaneous flap: Anatomic observations and report of 60 cases". Plastic and reconstructive surgery, 71.2: 199-204, 1983.

12- Demirtas Y., et al.: "Transaxillary-subclavian transfer of pedicled latissimus dorsi musculocutaneous flap to head and neck region". Journal of Craniofacial Surgery, 21.3: 771-5, 2010.
13- Watson J.S., et al.: "Pharyngeal reconstruction using the latissimus dorsi myocutaneous flap". British Journal of Plastic Surgery, 35.4: 401-7, 1982

14- Hayden A., Richard E., Simon D. and Daniel G.: "Technical modifications of the latissimus dorsi pedicled flap to increase versatility and viability". The Laryngoscope, 110.3: 352-7, 2000.

15- Har-El G., Mahesh B. and Krishnamurthi S.: "Latissimus dorsi myocutaneous flap for secondary head and neck reconstruction". American Journal of Otolaryngology, 20.5: 287-93, 1999.

16- Paolini G., et al.: "Permanent latissimus dorsi muscle denervation in breast reconstruction". Annals of Plastic Surgery, 71.6: 639-42, 2013.

17- Demirtas Y., et al.: "Transaxillary-subclavian transfer of pedicled latissimus dorsi musculocutaneous flap to head and neck region". Journal of Craniofacial Surgery, 21.3: 771-5, 2010. 